\title{
Análisis y mejora de la adherencia terapéutica en una farmacia comunitaria de Ourense
}

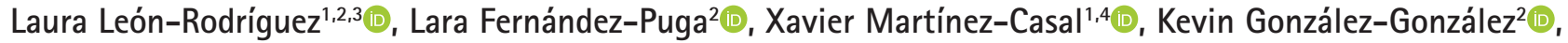 \\ $\mathrm{M}^{\mathrm{a}}$ Teresa Rodríguez-Rodríguez ${ }^{2}$, José Antonio Fornos-Pérez ${ }^{1,3,5}$, N. Floro Andrés-Rodríguez ${ }^{1,3}$ (1) \\ 1. Doctor en Farmacia. 2. Farmacéutico comunitario en Ourense. 3. Grupo Berbés de Investigación y Docencia. 4. Farmacéutico especialista \\ en Farmacia Hospitalaria. Servicio de Farmacia del Hospital Público de Cee (A Coruña). 5. Farmacéutico comunitario en Cangas do Morrazo \\ (Pontevedra).
}

PALABRAS CLAVE

Adherencia terapéutica, Servicios profesionales farmacéuticos, Farmacia comunitaria, Revisión del uso de los medicamentos (RUM), Sistema personalizado de dosificación (SPD), Satisfacción del paciente

\section{ABREVIATURAS}

DM: diabetes mellitus

HTA: hipertensión arterial

PRM: problemas relacionados con los medicamentos

RNM: resultados negativos de la medicación

RUM: revisión del uso de los medicamentos

SPD: sistema personalizado de dosificación

SPF: servicios profesionales farmacéuticos

\section{RESUMEN}

Objetivos: evaluar la adherencia terapéutica de pacientes con patologías prevalentes, identificando factores relacionados. Cuantificar la mejora en la adherencia conseguida mediante servicios profesionales farmacéuticos (SPF). Comprobar la satisfacción con el servicio.

Métodos: diseño: 1a fase: estudio monocéntrico, descriptivo, longitudinal, realizado entre 18/3 y 10/4/2019. 2a fase: estudio cuasiexperimental sin grupo control, con intervención educativa y SPF, revisión del uso de la medicación (RUM) y sistemas personalizados de dosificación (SPD), octubre-noviembre/2019.

Sujetos: pacientes mayores de edad con dislipemia, hipertensión o diabetes, que acudieron a la farmacia y aceptaron participar. Para la $2^{\text {a }}$ fase se seleccionaron pacientes que resultaron no adherentes en la $1^{\text {a. }}$.

Variables: adherencia (medida con el test Morisky-Green), satisfacción con el servicio. Variables demográficas.

Resultados: $1^{\text {a }}$ fase: 101 pacientes, media de edad de 69 años. 50,5\% hombres. La proporción de no adherentes fue del 55,4\%. 40 (71,4\%) olvidan alguna vez tomar sus medicamentos. La adherencia no resultó relacionada con sexo, edad, acompañamiento, número de patologías o nivel de estudios $(p>0,05)$.

2a fase: RUM: el porcentaje de no adherentes disminuyó al 67\%. SPD: 9 de los 10 pacientes resultaron adherentes al final del estudio. 1 paciente resultó no adherente por olvidar esporádicamente alguna toma.

El 100\% de las encuestas de satisfacción dio como resultado "muy satisfechos", continuarían visitando la farmacia y la recomendarian.

Conclusiones: la adherencia terapéutica resulto baja, menor del 50\%. No se encontró relación con los factores analizados.

La realización de RUM y SPD mejora la adherencia. El grado de satisfacción fue del $100 \%$. Todos los pacientes quisieron continuar con el servicio.

\section{INTRODUCCIÓN}

El aumento en la esperanza de vida unido al envejecimiento, ha provocado un incremento en el número de enfermedades crónicas, lo que lleva asociado un aumento en la prescripción y consumo de fármacos para el tratamiento y prevención de estas enfermedades. Por todo esto es habitual encontrar en la farmacia comunitaria numerosos pacientes con varias patologías. La polimedicación suele llevar asociada una disminución en la adherencia al tratamiento, la cual se ve influida también porque muchos pacientes no toman correctamente su medicación debido a diversas causas como son olvidos, falta de comprensión de la posología del tratamiento, dificultades en el manejo de dispositivos, etc. (1).

Los resultados de este estudio formaron parte de los Trabajos de Fin de Grado de Farmacia de Lara Fernández-Puga y Kevin González-González realizados en la farmacia de $\mathrm{M}^{\mathrm{a}}$ Teresa Rodríguez-Rodríguez en Ourense (Galicia).

Recibido: 09/12/2020

Aceptado: 24/03/202

Disponible online: 02/07/202

\author{
Financiación: ninguna. \\ Conflicto de intereses: ninguno. \\ Cite este artículo como: León-Rodríguez L, Fernández-Puga L, Martínez-Casal X, González-González K, Rodríguez-Ro- \\ dríguez MT, Fornos-Pérez JA, Andrés-Rodríguez NF. Análisis y mejora de la adherencia terapéutica en una farmacia \\ comunitaria de Ourense. Farmacéuticos Comunitarios. 2021 Jul 02: 13 (3): 5-16. doi:10.33620/FC.2173-9218.(2021/ \\ Vol13).003.02 \\ Correspondencia: Laura León-Rodríquez (lauralrb@hotmail.com). \\ ISSN 1885-8619 OSEFAC (Sociedad Española de Farmacia Clínica, Familiar y Comunitaria). Todos los derechos reservados.
}


La falta de adherencia supone un problema tanto para el paciente como para el sistema sanitario. En referencia al paciente, provoca una falta de mejoría clínica y una menor calidad de vida y a nivel social causa un mayor número de ingresos y un mayor gasto (2).

Es responsabilidad del farmacéutico comunitario asegurarse de que los pacientes, al retirar su medicación, sepan exactamente para qué la tienen que tomar y cómo tomarla, evitando así problemas relacionados con el medicamento (PRM) que conduzcan a resultados negativos de la medicación (RNM) (3).

En la actualidad, se considera que la actividad profesional del farmacéutico debe orientarse a la provisión de servicios profesionales farmacéuticos (SPF) $(4,5)$. Los SPF se dividen en dos grupos, dependiendo si están orientados a mejorar el proceso de uso de los medicamentos, o si están orientados a mejorar los resultados de la farmacoterapia. Entre los primeros se encuentra el servicio específico de adherencia terapéutica, o el de revisión del uso de la medicación (RUM) $(5,6)$, que incluye una evaluación del grado de adherencia del paciente. Consiste en la realización por parte del farmacéutico, junto con el paciente y el cuidador, de una revisión estructurada del grado de conocimiento que este tiene de sus medicamentos y del uso que hace de los mismos, ofreciendo asesoramiento sobre su utilización correcta y asegurándose de que el paciente entiende qué toma, cómo lo toma, para qué lo toma y durante cuánto tiempo lo tiene que tomar (7).

Una de las herramientas más utilizadas para mejorar la adherencia en pacientes ancianos crónicos, polimedicados y/o con dificultades para tomar la medicación son los sistemas personalizados de dosificación (SPD). Se trata de sistemas de reacondicionamiento post-dispensación, en los que el farmacéutico prepara la medicación del paciente según la pauta prescrita por el médico prescriptor autorizado en el Real Decreto Ley 9/2011 (8) y protocolizado en el Documento de Buenas prácticas en farmacia comunitaria del Consejo General de Colegios Oficiales de Farmacéuticos (9).

El municipio de Ourense (Galicia) está constituido por una población de 107.597 habitantes, con una edad media de 46,79 años, siendo los mayores de 65 años el 25,2\% del total (10). La esperanza media de vida en Ourense es de 82,3 años (10). Las patologías más habituales corresponden a la hipertensión arterial (HTA), con un 13,7\% de prevalencia en la población comprendida entre los 45 y 64 años y el 30,8\% en los mayores de 65 años, mientras que el 17,0\% de la población de entre 45 y 64 años, y el 32,2\% de mayores de 65 años sufre dislipemia. La diabetes mellitus (DM) afecta a un 7,5\% de la población orensana (11). Además, el 39,3\% presenta sobrepeso y el 11,3\% obesidad, tratándose de una población sedentaria, ya que el $31 \%$ de la población reconoce no realizar ningún tipo de ejercicio físico (11).
Según un amplio estudio a nivel nacional sobre adherencia del Observatorio de la Adherencia al Tratamiento (OAT), el porcentaje de cumplimiento no llega en las enfermedades crónicas al $50 \%$. Las patologías donde se encontró menor adherencia fueron: la enfermedad cardiovascular con incumplimientos del 56,8\%, la diabetes con el $54,0 \%$ y la hipertensión, con el 53,5\%. Su coste se estimó en 11.250 millones de euros anuales (12). Se trata pues de un problema de gran importancia sanitaria, social y económica, en el que la farmacia comunitaria puede tener un papel fundamental por su proximidad y accesibilidad para el paciente y la continuidad en la atención que le presta, que no está limitada por citas previas y en la que incluso en tiempos de pandemia no ha dejado de ser atendido presencialmente.

La finalidad de este trabajo es conocer la adherencia en los pacientes con HTA, dislipemias y/o diabetes que acuden a una farmacia comunitaria de Ourense, y evaluar el impacto de la RUM y el uso de SPD en la mejora de la adherencia en estos pacientes.

\section{OBJETIVOS}

\section{General}

- Analizar la adherencia terapéutica y evaluar su mejora mediante la prestación de servicios profesionales farmacéuticos.

\section{Específicos}

- Evaluar la adherencia terapéutica de pacientes que presentan alguna patología prevalente.

- Identificar factores relacionados.

- Cuantificar la mejora en la adherencia al tratamiento conseguida.

- Comprobar la satisfacción de los pacientes con dichos servicios.

\section{MATERIAL Y MÉTODOS}

\section{Diseño}

El trabajo constó de dos partes. La primera fue un estudio monocéntrico, descriptivo, transversal en una farmacia comunitaria de Ourense realizado entre el 18 marzo y 10 abril 2019. La segunda parte fue un estudio cuasiexperimental sin grupo control, con intervención educativa y mediante SPF desarrollado en la misma farmacia en los meses de octubre y noviembre de 2019 (figura 1). 


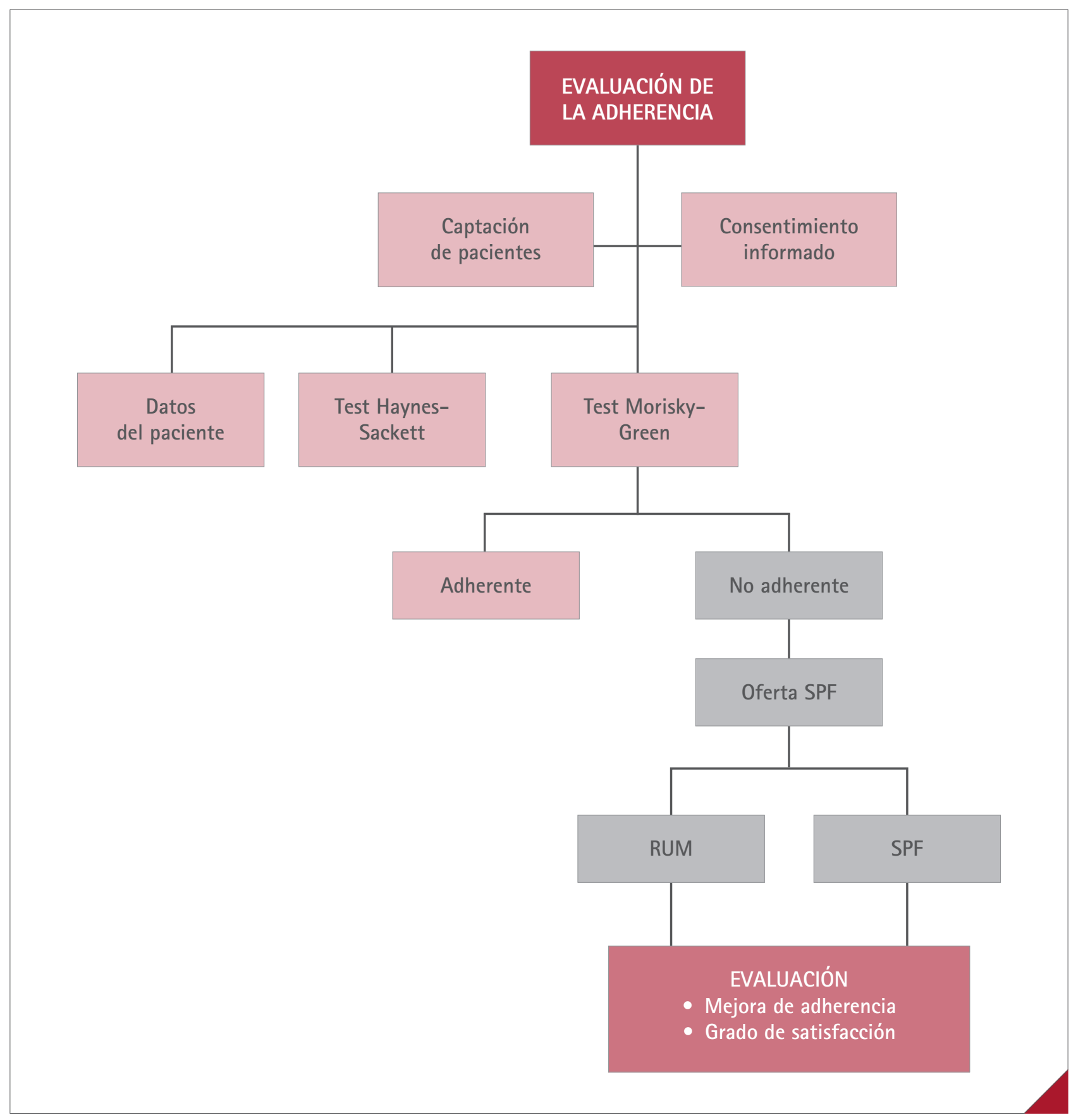

Figura 1 Esquema del estudio

\section{Sujetos}

Para la primera parte del estudio se seleccionaron todos los pacientes mayores de edad con dislipemia, hipertensión o diabetes, que acudieron a la farmacia en el periodo de estudio y aceptaron participar. Para la segunda se incluyeron pacientes de la farmacia entre 40 y 95 años, con dislipemia, hipertensión o diabetes, que habian resultado no adherentes en el estudio sobre adherencia previo.

\section{Criterio de exclusión}

Negativa a participar.

\section{Variables}

Variables principales

Adherencia ( $\%$ de adherentes), test de Haynes-Sackett $(13)$ y test de Morisky-Green $(14,15)$. Se consideraron no adherentes los pacientes que fallaron alguna de las 4 preguntas. Grado de satisfacción con el servicio: cuestionario ad hoc, escala Likert, basado en el de Armando (16) (figura 2).

Variables secundarias

Sexo, edad, nivel de estudios, vivir solo, número de medicamentos, hábito tabáquico, ejercicio físico. 


\begin{tabular}{|c|c|c|c|c|c|}
\hline $\begin{array}{l}\text { Las veces que acudí a la farmacia para que } \\
\text { me presten sus servicios }\end{array}$ & $\begin{array}{l}\text { Totalmente en } \\
\text { desacuerdo }\end{array}$ & $\begin{array}{c}\text { En } \\
\text { desacuerdo }\end{array}$ & $\begin{array}{l}\text { Ni de acuerdo } \\
\text { ni en desacuerdo }\end{array}$ & $\begin{array}{l}\text { De } \\
\text { acuerdo }\end{array}$ & $\begin{array}{l}\text { Muy de } \\
\text { acuerdo }\end{array}$ \\
\hline \multicolumn{6}{|l|}{$\begin{array}{l}\text { El personal se mostró interesado en } \\
\text { colaborar conmigo en el manejo de mis } \\
\text { medicamentos }\end{array}$} \\
\hline \multicolumn{6}{|l|}{$\begin{array}{l}\text { El tiempo dedicado por el personal fue el } \\
\text { adecuado }\end{array}$} \\
\hline \multicolumn{6}{|l|}{ Fueron amables durante la atención } \\
\hline \multicolumn{6}{|l|}{$\begin{array}{l}\text { Recibí la información adecuada sobre } \\
\text { cómo utilizar mis medicamentos }\end{array}$} \\
\hline \multicolumn{6}{|l|}{$\begin{array}{l}\text { Me informaron de los efectos secundarios } \\
\text { más frecuentes de los medicamentos que } \\
\text { uso }\end{array}$} \\
\hline \multicolumn{6}{|l|}{$\begin{array}{l}\text { Me transmitieron confianza al darme sus } \\
\text { recomendaciones }\end{array}$} \\
\hline $\begin{array}{l}\text { Como consecuencia de los servicios } \\
\text { prestados por la farmacia }\end{array}$ & $\begin{array}{l}\text { Totalmente en } \\
\text { desacuerdo }\end{array}$ & $\begin{array}{c}\text { En } \\
\text { desacuerdo }\end{array}$ & $\begin{array}{l}\text { Ni de acuerdo } \\
\text { ni en desacuerdo }\end{array}$ & $\begin{array}{c}\text { De } \\
\text { acuerdo }\end{array}$ & $\begin{array}{l}\text { Muy de } \\
\text { acuerdo }\end{array}$ \\
\hline \multicolumn{6}{|l|}{$\begin{array}{l}\text { Aprendí a conocer mejor los } \\
\text { medicamentos que uso }\end{array}$} \\
\hline \multicolumn{6}{|l|}{$\begin{array}{l}\text { Continuaría visitando esta farmacia para } \\
\text { que me brinden sus servicios profesionales }\end{array}$} \\
\hline \multicolumn{6}{|l|}{$\begin{array}{l}\text { Recomendaria a mis familiares o amigos } \\
\text { que acudan a esta farmacia para que se } \\
\text { les brinden estos servicios }\end{array}$} \\
\hline Estoy satisfecho con los servicios recibidos & & & & & \\
\hline
\end{tabular}

Figura 2 Cuestionario de satisfacción utilizado en el estudio

\section{Procedimiento}

Según criterios basados en el tipo de no adherencia y dificultad que presentaba cada paciente a la hora de no cumplir con su tratamiento, se derivaron a los pacientes a los distintos servicios profesionales. Los pacientes que tenían problemas o dudas en cuanto a la posología, indicación o duración del tratamiento, fueron derivados a RUM y los pacientes con dificultades a la hora de manejar su medicación fueron trasladados a SPD: se utilizaron SPD manuales elaborados integramente por un farmacéutico acreditado, responsable del servicio de SPD, tanto a nivel asistencial como técnico, y supervisado por otro farmacéutico. Estos sistemas presentan la ventaja de ser más económicos y la desventaja de requerir más tiempo de preparación y personal (17). Los SPD se prepararon semanalmente y eran recogidos por el paciente o el cuidador una vez a la semana. Cuando entregaban el blister de la semana anterior se comprobaba si se había tomado toda la medicación o si quedaba alguna sin tomar. En la recogida de los blísteres se controló la medicación no utilizada. Al cabo de 8 semanas se evaluó de nuevo la adherencia y se administró el cuestionario de satisfacción.

En Anexos se presentan los documentos de información al paciente, consentimiento informado y hoja de registro de datos. 


\section{Tamaño muestral}

Para una precisión del 10,0\% en la estimación de una proporción mediante intervalo de confianza asintótico Normal al 95\% bilateral, asumiendo una proporción del 50,0\% y que la población relacionada con la farmacia se estimó en 28.500 personas (las que acuden al centro de salud próximo), resultó necesario incluir 96 pacientes. Para la segunda parte, debido al sistema de inclusión de los pacientes en el estudio (procedentes de la evaluación previa) no se realizó el cálculo del tamaño muestral.

\section{Análisis estadístico}

Las variables cuantitativas se expresan como media $(\mathrm{m}) \pm$ desviación estándar (DE), las cualitativas como porcentajes. Para la recogida, explotación y análisis de los datos se utilizaron el paquete ofimático Microsoft Exce ${ }^{\circledR} 2011$ y el paquete estadistico IBM SPSS Statistics ${ }^{\circledR}$ versión 20.0 (IBM Corporation ${ }^{\circledR}$ ) para $\mathrm{Mac}^{\circledR}$. Para variables cuantitativas con distribución normal se utilizó la t-student de muestras independientes y Anova de un factor ( $>2$ clases). En caso de variable categórica se usó Chi-cuadrado de Pearson. En caso de variable cuantitativa no paramétrica para muestras no relacionadas se utilizó la Zkolmogorov-Smirnov. Para variables categóricas en muestras no relacionadas se usó de Chi-cuadrado de Pearson. Se consideró un nivel de significación estadistica de $p<0,05$ estableciéndose el valor de las diferencias obtenidas con su intervalo de confianza al 95\%.

\section{Aspectos éticos}

Se respetó en todos los casos la autonomía de los participantes en la investigación, siguiendo los principios éticos de la Declaración de Helsinki vigente y la Ley Orgánica 3/2018, de 5 de diciembre, de Protección de Datos Personales y garantía de los derechos digitales. Los datos se registraron en una tabla de forma anonimizada. Previamente se proporcionó a los pacientes la información pertinente y se recogió el consentimiento informado en todos los casos.

\section{RESULTADOS}

\section{Determinación de la adherencia al tratamiento}

En la primera parte del estudio se propuso participar a 110 pacientes. 9 de ellos decidieron no aceptar por falta de tiempo, por lo que el número de participantes fue de 101. La media de edad fue de 69 años. El 28,7\% (29) entre 40-64 años; $32,7 \%$ (33) entre 65-74; 29,7\% (30) entre 75-84, y 8,9\% (9) más de 85 años. El 50,5\% hombres. El 31,7\% (32) presentaba una de las patologías a estudiar, el 52,5\% (53) dos patologías y $15,8 \%$ (16) las tres. En la tabla 1 se presentan las diferentes patologias en función del rango de edad. El 6,9\% (7) eran fumadores, el 29,7\% (30) reconoció no practicar casi ninguna actividad física, el 50,5\% (51) decía realizar actividad física de 6-10 horas semanales y el 19,8\% (20), 11-20 horas.

Según el método indirecto de autocomunicación de cumplimiento de Haynes-Sackett, el $99 \%$ de los pacientes encuestados contestaron no tener dificultades en tomar sus medicamentos. Según el test Morisky-Green, la proporción de adherentes fue del $44,6 \%$ (45 pacientes).

De los 56 pacientes $(55,4 \%)$ no adherentes al tratamiento, $40(71,4 \%)$ reconocieron olvidarse alguna vez de tomar los medicamentos para tratar la enfermedad. El 39,6\% (37) del total resultaron no adherentes por olvidos en la toma de la medicación, mientras que el 15,8\% (16) restante de los pacientes fueron no adherentes por otras causas.

Tabla 1 Prevalencia de las diferentes patologias agrupadas por edad de pacientes

\begin{tabular}{|c|c|c|c|c|c|}
\hline Patologías & $\begin{array}{c}\text { 40-64 años } \\
\text { n (\%) }\end{array}$ & $\begin{array}{c}\text { 65-74 años } \\
n(\%)\end{array}$ & $\begin{array}{c}\text { 75-84 años } \\
\text { n (\%) }\end{array}$ & $\begin{array}{c}>85 \text { años } \\
\text { n (\%) }\end{array}$ & $\begin{array}{l}\text { Total } \\
\text { n (\%) }\end{array}$ \\
\hline DM & $3(75,0)$ & $1(25,0)$ & 0 & 0 & $4(100)$ \\
\hline HTA & $8(47,1)$ & $2(11,8)$ & $7(41,2)$ & 0 & $17(100)$ \\
\hline Dislipemia & $7(58,3)$ & $1(8,3)$ & $2(16,7)$ & $2(16,7)$ & $12(100)$ \\
\hline Dislipemia+HTA & $6(16,2)$ & $17(46,0)$ & $10(27,0)$ & $4(10,8)$ & $37(100)$ \\
\hline $\mathrm{HTA}+\mathrm{DM}$ & $1(11,1)$ & 0 & $6(66,7)$ & $2(22,2)$ & $9(100)$ \\
\hline Dislipemia+DM & $1(14,3)$ & $3(42,9)$ & $3(42,9)$ & 0 & $7(100)$ \\
\hline Dislipemia+DM+HTA & $3(20,0)$ & $9(60,0)$ & $2(13,3)$ & $1(6,7)$ & $15(100)$ \\
\hline Total & $29(28,7)$ & $33(32,67)$ & $30(29,71)$ & $9(8,9)$ & $101(100)$ \\
\hline
\end{tabular}

DM: diabetes mellitus; HTA: hipertensión arterial. 
Tabla 2 Resultados para cada item del test Morisky-Green

\begin{tabular}{|l|c|c|}
\hline & $\begin{array}{c}\text { Sí } \\
\mathrm{n}(\%)\end{array}$ & $\begin{array}{c}\text { No } \\
\mathrm{n}(\%)\end{array}$ \\
\hline $\begin{array}{l}\text { ¿Olvida alguna vez tomar los } \\
\text { medicamentos para tratar su } \\
\text { enfermedad? }\end{array}$ & $39(38,6)$ & $62(61,4)$ \\
\hline $\begin{array}{l}\text { ¿Toma los medicamentos a las } \\
\text { horas indicadas? }\end{array}$ & $71(70,3)$ & $30(29,7)$ \\
\hline $\begin{array}{l}\text { Cuando se encuentra bien, ¿deja } \\
\text { de tomar la medicación? }\end{array}$ & $1(1,0)$ & $100(99,0)$ \\
\hline $\begin{array}{l}\text { ¿Alguna vez deja de tomar sus } \\
\text { fármacos o toma menos si le } \\
\text { sientan mal? }\end{array}$ & $0(0,0)$ & $101(100)$ \\
\hline
\end{tabular}

En la tabla 2 se presentan los resultados para cada ítem del test Morisky-Green.

El grado de adherencia no resultó directamente relacionado con sexo, edad, acompañamiento, número de patologías, nivel de estudios, hábito tabáquico y actividad física de los pacientes $(p>0,05)$ (tabla 3$)$.

\section{Mejora de la adherencia mediante SPF}

En la segunda parte del trabajo, a los pacientes no adherentes se les propuso la derivación a alguno de los servicios farmacéuticos profesionales, 40 pacientes consideraron no necesitarlo y no continuaron, 6 pacientes no adherentes se derivaron al RUM y 10 se derivaron a SPD siguiendo los criterios descritos en la metodología.

Tabla 3 Relación de la adherencia al tratamiento con factores sociodemográficos y clínicos

\begin{tabular}{|c|c|c|c|c|}
\hline Factores & $\begin{array}{c}\text { Adherentes } \\
\text { n (\%) }\end{array}$ & $\begin{array}{c}\text { No adherentes } \\
n(\%)\end{array}$ & $\begin{array}{l}\text { Total } \\
\mathrm{n}(\%)\end{array}$ & $\mathrm{p}$-valor \\
\hline \multicolumn{5}{|l|}{ Hábito tabáquico } \\
\hline $\begin{array}{l}\text { No fumador } \\
\text { Fumador }\end{array}$ & $\begin{array}{c}42(41,6) \\
3(3,0)\end{array}$ & $\begin{aligned} 52 & (51,5) \\
4 & (4,0)\end{aligned}$ & $\begin{array}{c}94(93,1) \\
7(6,9)\end{array}$ & $0,925^{*}$ \\
\hline \multicolumn{5}{|l|}{ Sexo } \\
\hline $\begin{array}{l}\text { Mujer } \\
\text { Hombre }\end{array}$ & $\begin{array}{l}23(22,7) \\
22(21,8)\end{array}$ & $\begin{array}{l}27(26,7) \\
29(28,7)\end{array}$ & $\begin{array}{l}45(44,6) \\
56(55,5)\end{array}$ & $0,772^{*}$ \\
\hline \multicolumn{5}{|l|}{ Acompañamiento } \\
\hline $\begin{array}{l}\text { Vivir solo } \\
\text { Vivir acompañado }\end{array}$ & $\begin{array}{c}5(5,0) \\
40(39,6)\end{array}$ & $\begin{array}{l}13(12,9) \\
43(42,6)\end{array}$ & $\begin{array}{l}18(17,8) \\
83(82,2)\end{array}$ & $0,114^{*}$ \\
\hline \multicolumn{5}{|l|}{ № patologías } \\
\hline $\begin{array}{l}1 \\
2 \\
3\end{array}$ & $\begin{array}{c}14(13,9) \\
24(23,8) \\
7(6,9)\end{array}$ & $\begin{array}{c}18(17,8) \\
29(28,7) \\
9(8,9)\end{array}$ & $\begin{array}{l}32(31,7) \\
53(52,5) \\
16(15,8)\end{array}$ & $0,988^{* *}$ \\
\hline \multicolumn{5}{|l|}{ Edad } \\
\hline $\begin{array}{l}\text { 40-64 años } \\
65-74 \text { años } \\
75-84 \text { años } \\
>85 \text { años }\end{array}$ & $\begin{array}{c}14(13,9) \\
17(16,8) \\
10(9,9) \\
4(4,0)\end{array}$ & $\begin{array}{c}15(14,9) \\
16(15,8) \\
20(19,8) \\
5(5,0)\end{array}$ & $\begin{array}{c}29(28,7) \\
33(32,7) \\
30(29,7) \\
9(8,9)\end{array}$ & $0,505^{* *}$ \\
\hline \multicolumn{5}{|l|}{ Nivel de estudios } \\
\hline $\begin{array}{l}\text { Primarios } \\
\text { Secundarios } \\
\text { Superiores }\end{array}$ & $\begin{array}{c}20(19,8) \\
21(20,8) \\
4(4,0)\end{array}$ & $\begin{array}{c}21(20,8) \\
29(28,7) \\
6(5,9)\end{array}$ & $\begin{array}{c}41(40,6) \\
50(49,5) \\
10(9,9)\end{array}$ & $0,774^{* *}$ \\
\hline \multicolumn{5}{|l|}{ Nivel de actividad } \\
\hline $\begin{array}{l}\text { Bajo (0-5 h/semana) } \\
\text { Medio (6-10 h/semana) } \\
\text { Alto (11-20 h/semana) }\end{array}$ & $\begin{array}{c}11(10,9) \\
24(23,8) \\
10(9,9)\end{array}$ & $\begin{array}{c}19(18,8) \\
27(26,7) \\
10(9,9)\end{array}$ & $\begin{array}{l}30(29,7) \\
51(50,5) \\
20(19,8)\end{array}$ & $0,57^{* *}$ \\
\hline
\end{tabular}

* Test Chi-cuadrado de Pearson.

** Test Anova de un factor. 
De los pacientes derivados a RUM, el porcentaje de no adherentes disminuyó del 100\% al 66,7\% (4 todavía olvidaban en alguna ocasión la toma de la medicación).

9 de los 10 pacientes incluidos en SPD resultaron adherentes al final del estudio. Solamente 1 paciente resultó no adherente por incumplimiento esporádico al olvidar alguna toma.

Todos los blísteres que los pacientes devolvían a la farmacia, venían con la medicación consumida. El $90 \%$ de los pacientes (9) recogía la medicación el día que les correspondía, pero uno $(10 \%)$ la recogió sistemáticamente dos días tarde, ya que hasta en dos ocasiones, el paciente se olvidó tomar la medicación.

El $100 \%$ de las encuestas de satisfacción dio como resultado estar "muy de acuerdo" en todas las preguntas realizadas, manifestando los pacientes estar muy satisfechos con los servicios recibidos, como consecuencia de los cuales aprendieron a conocer mejor la medicación, continuarían visitando la farmacia y la recomendarian a familiares y amigos.

\section{DISCUSIÓN}

\section{Análisis de la adherencia}

La adherencia farmacológica fue diferente, dependiendo del test utilizado, con el test de Haynes-Sackett fue del $99 \%$ y con el Morisky-Green 44,6\%, en la línea de otros estudios publicados $(12,18,19)$. Las diferencias entre ambos se deben a que cada uno aborda la evaluación de la adherencia desde un aspecto diferente. El test de Haynes-Sackett es utilizado en ocasiones para ambientar al paciente entrevistado, y que se sienta más cómodo cuando responda al otro test (20), ya que su utilidad es limitada por su baja sensibilidad. El de Morisky-Green mide la adherencia desde el comportamiento que tiene la persona hacia la toma de medicación (21). En nuestro caso indica que el olvido de la toma es la principal causa de incumplimiento.

El estudio se basa en la recogida de información procedente de los propios pacientes y ha de tenerse en cuenta que los resultados obtenidos en este estudio se han obtenido aplicando métodos indirectos de cumplimiento autocomunicado, que tienen como inconveniente que sobrestiman la adherencia, ya que existe la posibilidad de que el paciente exagere, olvide datos sobre la toma de medicación o que piense que hace correctamente el tratamiento, pero no sea así (falta de adherencia involuntaria) (15).

Otra limitación del estudio es la pequeña muestra que participó en la segunda parte, que vino determinada por tratarse de un estudio monocéntrico, condicionado por el número de pacientes que aceptaron participar, ya que en su gran mayoria consideraron que no necesitaban el servicio ofrecido. Debería hacerse un esfuerzo en dar a conocer los SPF que la farmacia comunitaria puede poner a disposición de los pacientes.
En nuestro estudio, de las 45 personas que presentan adherencia al tratamiento 23 son mujeres y 22 son hombres, y la prueba estadística indica que ambos sexos mostraron igual comportamiento frente a la toma de medicación, coincidiendo con algunos estudios $(1,22)$ pero no con otros que si muestran una mayor adherencia en el sexo femenino que en el masculino (23) o viceversa (19).

Tampoco en nuestra muestra se encontraron diferencias significativas en cuanto a la edad, al igual que describe Leites-Docío et al (22), pero diferente a otros estudios donde sí que se encontraron diferencias significativas en la adherencia con mejor cumplimiento a mayor edad del tratamiento en pacientes diabéticos e hipertensos $(24,25)$.

A pesar de que en otros estudios (19) la adherencia se ve disminuida con niveles de estudios bajos, en nuestro trabajo esta no se ve afectada, al igual que describe Leites-Docio et al (22), lo que podría estar justificado por el diferente cuestionario utilizado para medir la adherencia.

Otro factor evaluado fue si vivir solo o acompañado podía influir en la adherencia al tratamiento. Se eligió este factor, ya que en muchas ocasiones es algún cuidador o familiar el que recoge la medicación y el que le recuerda al paciente que debe tomar su medicación. En nuestro estudio el hecho de vivir solo o acompañado no es un factor estadisticamente significativo en la adherencia. Por otro lado, tampoco se encontró relación entre el número de patologías con la adherencia. Se trata de un factor controvertido en el que coincidimos con algunos estudios $(20,22)$, pero no con otros $(1,19,26)$.

Como hemos visto no hay una coincidencia entre los diversos estudios sobre la posible relación entre los factores que se supone podrian tener influencia en la adherencia terapéutica y los resultados de los cuestionarios que la evalúan. Tal vez diferencias metodológicas o de entorno puedan explicar esta disparidad de resultados.

\section{Mejora de la adherencia terapéutica}

En la segunda fase del estudio, independientemente del SPF prestado, la mejora de la adherencia fue notable, lo que indica de manera clara, aun al modesto nivel que supone una única farmacia comunitaria, que, tal como encontramos en otros trabajos revisados, la intervención del farmacéutico, mediante diversos métodos, mejora significativamente el cumplimiento por parte del paciente de su tratamiento farmacoterapéutico, bien sea mediante seguimiento específico con intervención educativa (27), RUM (28) u otros modelos de intervención (29-31).

El hecho de que haya un número muy elevado de pacientes no adherentes, tal y como quedó demostrado en el estudio, nos permitió comprobar que los servicios profesionales que ofrecen algunas farmacias son una herramienta muy importante tanto para los pacientes como para la Administración, ya que su uso generalizado permitiría una disminución en los costes sanitarios asociados al mal uso de 
los medicamentos, especialmente la falta de adherencia con el elevado coste social y económico que conlleva (32).

El grado de satisfacción de los pacientes fue muy alto, coincidiendo con otros trabajos de realización de SPF (33). A pesar de tratarse de una muestra pequeña, el estudio permitió comprobar que se produce una considerable mejora en el proceso de uso de los medicamentos, que sin duda repercutirá en la obtención de mejores resultados en su salud. En todo caso, la comunicación con el médico facilita la prestación del servicio y un uso más racional de la medicación.

Consideramos, por lo tanto, y a la vista de este alto grado de satisfacción obtenido mediante la prestación de los SPF implementados para mejorar la adherencia, que su extensión a un número importante de las farmacias comunitarias, en las dimensiones que las circunstancias de cada una lo permitieran, proporcionaría una herramienta excelente para mejorar la utilización de los tratamientos $y_{\text {, por }}$ lo tanto, el estado de salud de los pacientes que retiran de ellas su medicación.

\section{CONCLUSIONES}

Los pacientes de la farmacia donde se realizó el estudio presentan un bajo grado de adherencia terapéutica, menor del 50\%. No se encontró relación estadísticamente significativa con los factores analizados. Se necesitan acciones enfocadas a mejorar la adherencia.

A pesar del pequeño tamaño de la muestra, creemos que la realización de RUM y SPD logra una considerable mejora en la adherencia al tratamiento. La efectividad parece mayor en el caso del SPD.

El grado de satisfacción fue del $100 \%$ y todos los pacientes quisieron continuar con el servicio.

\section{REFERENCIAS BIBLIOGRÁFICAS}

1. Figueiredo D, Teixeira L, Poveda V, Paúl C, Santos-Silva A, Costa E. Predictors of Difficulty in Medication Intake in Europe: A Cross-country Analysis Based on SHARE. Aging and Disease 2016; 7(3):246-253. doi:10.14336/AD.2015.0925

2. Gavilán-Moral E, Villafaina-Barroso A, Jiménez de Gracia A, Gómez Santana MC. Ancianos frágiles polimedicados: ¿es la deprescripción de medicamentos la salida? Revista Española de Geriatría y Gerontología. 2012; 47(4):162-167. doi:10.1016/j.regg.2012.01.003

3. Consejo general de Colegios Oficiales de Farmacéuticos Buenas Prácticas en Farmacia Comunitaria en España. 2013 [Acceso 29/11/2020]. Disponible en: https://www.portalfarma.com/Profesionales/Buenas-practicas-profesionales/Documents/Buenas-Practicas-Profesionales.pdf

4. Foro de Atención Farmacéutica-Farmacia Comunitaria (Foro AF-FC). Guia práctica para los Servicios Profesionales Farmacéuticos Asistenciales en la Farmacia Comunitaria. Madrid: Consejo General de Colegios Oficiales de Farmacéuticos; 2019. Disponible en: https:// www.portalfarma.com/inicio/serviciosprofesionales/forofarmaciacomunitaria/Documents/2019-guia-practica-spfa.pdf

5. Baixauli VJ, Satué-de-Velasco E, Gil MI, Roig JC, Villasuso B, Sáenz-de-Buruaga S. Propuesta de la Sociedad Española de Farmacia Comunitaria (SEFAC) sobre servicios profesionales farmacéuticos en farmacia comunitaria. Farmacéuticos Comunitarios. 2013 Sep 01; 5(3):119-126. Disponible en: https://www.farmaceuticoscomunitarios.org/es/journal-article/propuesta-sociedad-espanola-farmacia-comunitaria-sefac-sobre-servicios-profesionales

6. Consejo General de Colegios Oficiales de Farmacéuticos. Servicios farmacéuticos: Definición y remuneración. 2013. [Acceso 29/11/2020]. Disponible en: http://www.portalfarma.com/profesionales/organizacionfcolegial/profesionfarma/planfuturofarmaciacomunitaria// Documents/Servicios\%20Farmaceuticos_mayo\%202013.pdf

7. Gómez-Bermúdez E, García-Agua-Soler N, Jódar-Sánchez F, Baixauli-Fernández VJ. Proyecto Revisa. Impacto del servicio de revisión del uso de los medicamentos en España. Informe de tiempo y costes. Farmacéuticos Comunitarios 2017 Sep 30; 9(3): 20-24. doi:10.5672/ FC.2173-9218.(2017/Vol9).003.05

8. Cortes Españolas. Real Decreto-ley 9/2011, de 19 de agosto, de medidas para la mejora de la calidad y cohesión del sistema nacional de salud, de contribución a la consolidación fiscal, y de elevación del importe máximo de los avales del Estado para 2011. BOE nº 200. 20 /8/2011:93143-93168. Disponible en: https:|/www.boe.es/eli/es/ $\mathrm{rdl} / 2011 / 08 / 19 / 9 / \mathrm{con}$

9. Consejo General de Colegios Oficiales de Farmacéuticos. Buenas Prácticas en Farmacia Comunitaria en España14. Servicio de elaboración y provisión de Sistemas Personalizados de Dosificación (SPD). 2018. [Acceso 29/11/2020]. Disponible en: https://www.portalfarma. com/Profesionales/Buenas-practicas-profesionales/Documents/ BBPP-14-SPD.pdf

10. Instituto Galego de Estadística. Indicadores de poboación ano 2019. Indicadores demográficos. [Acceso 27/11/2020]. Disponible en: https://www.ige.eu/web/mostrar_actividade_estatistica.jsp?idioma $=$ esetcodigo $=0201004$

11. Servicio Gallego de Salud. SERGAS: Salud Pública. II Plan Municipal de Salud de Ourense 2014-2018. [Acceso 28/11/2020]. Disponible en: https://www.sergas.es/Saude-publica/Documents/4714/Paz_Villar_San_Martin.pdf

12. Observatorio de Adherencia al Tratamiento. Investigación en adherencia. Estudio Nacional de Adherencia al Tratamiento. 2019. [Acceso 30/11/2020]. Disponible en: https://www.oatobservatorio.com/ wp-content/uploads/2019/11/02.PPT-Ernesto-Cort\%C3\%A9s.pdf

13. Haynes RB, McKibbon KA, Kanani R, Brouwers MC, Oliver T. Interventions for helping patients to follow prescriptions for medications (Cochrane Review). In: The Cochrane Library, Issue 1, 1999. Oxford: Update Software.

14. Morisky DE, Green LW, Levine DM. Concurrent and predictive validity of a self-reported measure of medication adherence. Med Care. 1986; 24:67-74. doi:10.1097/00005650-198601000-00007

15. Pagès-Puigdemont N, Valverde-Merino MI. Métodos para medir la adherencia terapeútica. Ars Pharm. 2018; 59(3):163-172. doi:10.30827/ars.v59i3.7387

16. Armando P. Desarrollo y validación de cuestionarios de satisfacción de pacientes con los servicios de atención farmacéutica en farmacias comunitarias. Tesis doctoral Granada: Facultad de Farmacia Universidad de Granada; 2007. Disponible en: https://hera.ugr.es/tesisugr/1676853x. pdf

17. Maestre Hernández AB, González Valdivieso M, Arasa J, López Pintor E. Estudio de Revisión de las ventajas e inconvenientes de los Sistemas Personalizados de Dosificación (SPD) y evaluación de su adecuación al perfil del paciente y tipo de farmacia. Farmajournal 2020; 5(1):47-54. doi:10.14201/fj2020514754 
18. Espinosa García J, Martell Claros N, Llerena Ruiz A, Fernández Bergés D. Cumplimiento farmacológico en el tratamiento de la hipertensión arterial. Revisión de los estudios publicados entre los años 1975 y 2011. Semergén. 2012; 38(5):292-300. doi:10.1016/j.semerg.2012.01.001

19. Fornos-Pérez JA, Andrés-Rodríguez NF, Andrés-Iglesias JC, Mera-Gallego R, Mera-Gallego I, Penín-Álvarez Ó, Brizuela-Rodicio L. Valoración del cumplimiento de los tratamientos hipoglucemiantes y antihipertensivos en Galicia (CumpleGa). Farmacéuticos Comunitarios 2017; 9(4):5-13. doi:10.5672/FC.2173-9218.(2017/Nol9).004.02

20. Parody Rua E, Vásquez Vera AF. Prevalencia y factores asociados a la adherencia en un centro de atención primaria de Cali: comparación de 3 test de adherencia. Pharm Care Esp. 2019; 21(1):23-40. Disponible en: https:// www.pharmcareesp.com/index.php/PharmaCARE/article/view/487/395

21. Rodríguez Chamorro MA, García Jiménez $E_{1}$ Rodríguez Pérez $A, B a-$ tanero Hernán C, Pérez Merino EM. Revisión de test validados para la valoración de la adherencia al tratamiento farmacológico utilizados en la práctica clínica habitual. Pharm Care Esp. 2020; 22(3): 148-172. Disponible en: https://www.pharmcareesp.com/index.php/ PharmaCARE/article/view/572/460

22. Leites-Docio A, Garcia-Rodriguez $P$, Fernández-Cordeiro, $M$, Tenorio-Salgueiro L, Fornos-Pérez JA, Andrés-Rodríguez NF. Evaluación de la no adherencia al tratamiento hipoglucemiante en la farmacia comunitaria. Farmacéuticos Comunitarios. 2019;11(1):5-13. doi:10.5672/FC.2173-9218.(2019/Nol11).001.02

23. Agámez Paternita AP, Hernández Riera R, Cervera Estrada L, Rodriguez García Y. Factores relacionados con la no adherencia al tratamiento antihipertensivo. Arch Méd Camagüey. 2008;12(5). Disponible en: http://revistaamc.sld.cu/index.php/amc/article/view/2506

24. Tunceli K, Iglay K, Zhao C, Brodovicz KG, Radican L. Factors associated with adherence to oral antihyperglicemic monotherapy in patients with type 2 diabetes mellitus in the United Kingdom. Diab Res Clin Pract. 2015; 109(3):27-31. doi:10.1016/j.diabres.2015.05.031

25. Choi HJ, Oh IJ, Lee JA, Lim J, Kim YS, Jeon TH, Factors affecting adherence to antihypertensive medication. Korean J Fam Med, 2018; 39(6):325-332. doi:10.4082/kjfm.17.0041
26. Lorido $\mathrm{Cl}$, Hernanz $\mathrm{AB}$, Cordero SE, González HA, Menaya MRG, Gómez EJ. Adherencia terapéutica en pacientes polimedicados y factores condicionantes en un grupo de pacientes de Badajoz, España. Arch Med Fam. 2019; 21(2):51-59. Disponible en: https://www. medigraphic.com/pdfs/medfam/amf-2019/amf192c.pdf

27. Andrés Iglesias JC, Andrés Rodríguez NF, Fornos Pérez JA. Mejora del cumplimiento con hipolipemiantes. Farmacéuticos Comunitarios 2009; 1(3):94-100. Disponible en: https://www.farmaceuticoscomunitarios.org/es/system/files/journals/95/articles/01-3-94-100.pdf

28. Messerli M, Blozik E, Vriends N, Hersberger KE. Impact of a community pharmacist-led medication review on medicines use in patients on polypharmacy - a prospective randomized controlled trial. BMC Health Services Research 2016; 16:145. doi:10.1186/s12913-0161384-8

29. Nieuwlaat R, Wilczynski N, Navarro T, Hobson N, Jeffery R, Keepanasseril $A$, et al. Interventions for enhancing medication adherence. Cochrane Database of Systematic Reviews. 2014; 11: CD000011. doi:10.1002/14651858.CD000011.pub4

30. Akinbosoye OE, Taitel MS, Grana J, Hill JWade RL. Improving Medication Adherence and Health Care Outcomes in a Commercial Population through a Community Pharmacy. Population Health Management 2016; 19(6):454-461. doi:10.1089/pop.2015.0176

31. Bueno J, Vega-Coca MD, Rodríguez-Pérez A, Toscano-Guzmán MD, Pérez-Guerrero C, Santos-Ramos B. Intervenciones para la mejora de la adherencia al tratamiento en pacientes pluripatológicos: resumen de revisiones sistemáticas. Aten Primaria. 2016; 48(2):121-130. doi:10.1016/j.aprim.2015.02.012

32. Sicras-Mainara A, Huerta A, Sánchez D, Navarro-Artieda R. Uso de recursos y costes asociados a la no adherencia al tratamiento con corticoides inhalados en el asma. Semergén 2018;44(1):13-22. doi:10.1016/j.semerg.2017.03.005

33. Moranta FX, Huarte J. Promoción de los servicios profesionales farmacéuticos. 'El farmacéutico que necesitas': carpas de salud 2015-2016. Farmacéuticos Comunitarios. 2018 Mar 30; 10(1):7-17. doi:10.5672/FC.2173-9218.(2018/Vol10).001.03 


\section{ANEXOS}

Anexo 1. Documento de información para el paciente

\section{ANÁLISIS DE LA ADHERENCIA AL TRATAMIENTO FARMACOTERAPÉUTICO EN PACIENTES POLIMEDICADOS DESDE UNA FARMACIA COMUNITARIA EN OURENSE}

\section{¿Qué se va a realizar?}

En este proyecto el equipo investigador va a utilizar tres cuestionarios para realizar un estudio de la adherencia al tratamiento farmacoterapéutico para pacientes con hipertensión arterial, diabetes y/o hipercolesterolemia, con el objetivo de conocer las caracteristicas de los pacientes, de la farmacia y poder colaborar desde la farmacia para le mejora de sus tratamientos, y de su estado de salud.

He leído el presente documento;

FIRMA, NOMBRE Y APELLIDOS

Ourense, a de . 20. 
Anexo 2. Documento de consentimiento para la participación en proyecto de investigación

\section{ANÁLISIS DE LA ADHERENCIA AL TRATAMIENTO FARMACOTERAPÉUTICO EN PACIENTES POLIMEDICADOS DESDE UNA FARMACIA COMUNITARIA EN OURENSE}

D./Dña.

Con DNI n

Libre y voluntariamente manifiesto:

- Que he sido informado suficientemente del objetivo de estudio.

- Que estoy de acuerdo y acepto participar en el mismo voluntariamente durante todo el proceso.

- Que se me ha informado de que los datos recogidos en este estudio serán tratados confidencialmente, aplicándose la legislación vigente de protección de datos de carácter personal (Ley Orgánica 15/2999, 13 de diciembre) y cualquier otra que resultara aplicable.

- Que, salvaguardando siempre mi derecho a la intimidad, acepto que los datos derivados de mi participación puedan ser utilizados para la divulgación científica.

- Cualquier hallazgo importante que pudiera ser un riesgo importante para mi salud, me será comunicado inmediatamente.

- Comprendo que no se van a utilizar mis datos personales para otros estudios diferentes sin mi consentimiento.

- Comprendo que puedo retirarme del estudio cuando quiera, sin tener que dar explicaciones y sin que esto repercuta en mis cuidados médicos.

Por tanto, doy mi conformidad y consentimiento para que se realice el estudio detallado con la ayuda que sea necesaria con la debida cualificación y especialización.

El interesado 
Anexo 3. Hoja de recogida de datos del paciente

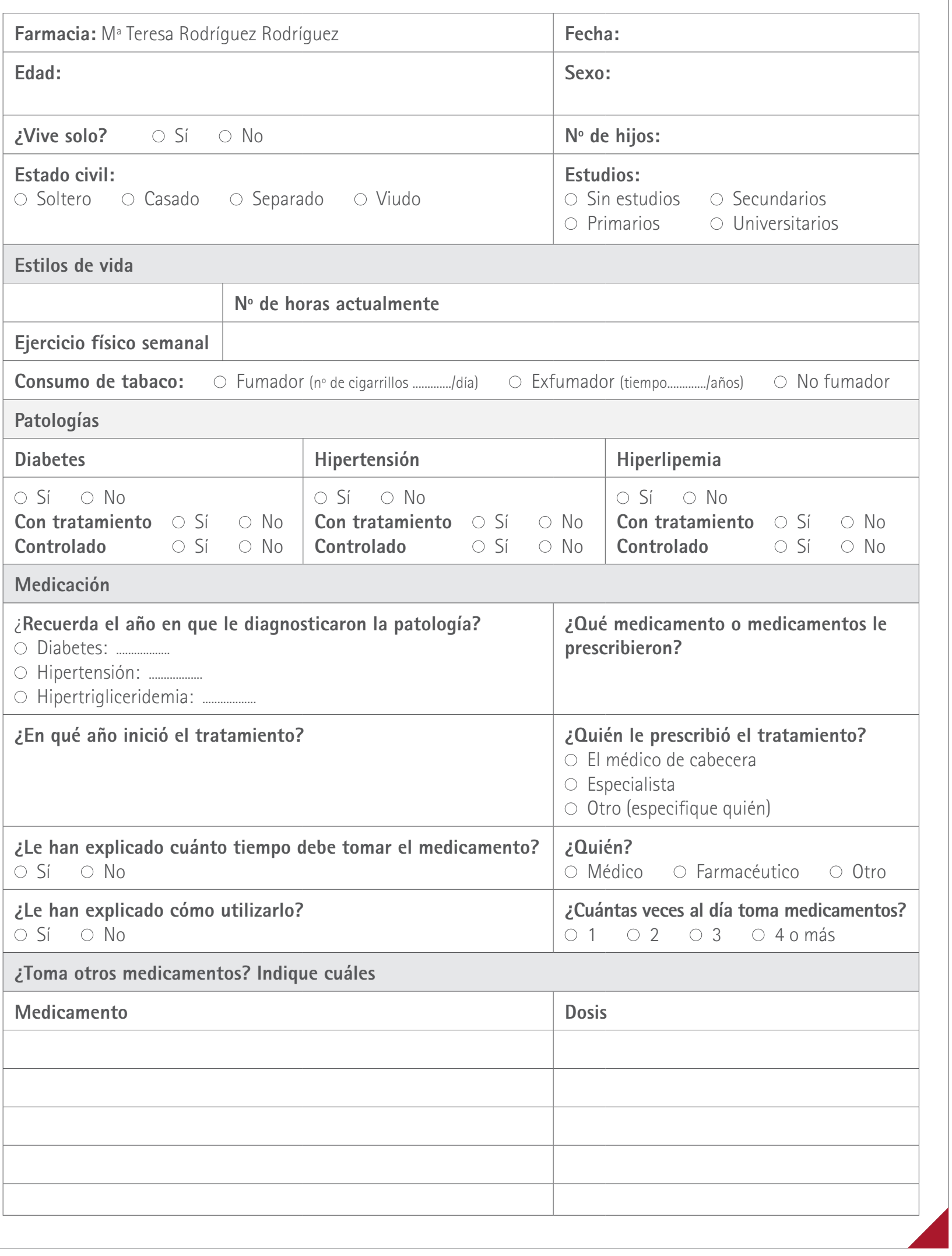

